\title{
Effect of Nutritional Intervention Treatment for Ovx Induced Osteoporosis on Oxidant-Antioxidant Status in Female Wistar Rats
}

\author{
Sakshi Siriah And Swati Kotwal* \\ University Department of Biochemistry RTM, Nagpur University, 440001
}

\begin{abstract}
Postmenopausal osteoporosis in women is the result of decline in synthesis of ovarian hormone estrogen, leading to reduced bone mass and increased bone turnover. Estrogen deficiency also affects antioxidant status in the body by production of reactive oxygen species. Continuous synthesis of reactive oxygen species damages the bone and enhances osteoclastogenesis. Irrespective of the therapy followed, Calcium, Vitamin D and Zinc are prescribed for osteoporosis. Osteoporosis being multifactorial disease in nature, only calcium and vitamin D supplement fails to make up for the bone loss. We addressed this problem by formulating treatments targeting various factors involved in the progression of osteoporosis by supplementing amino acids, genestein isoflavones and Vitamin K2. Increased oxidant status and decreased antioxidants enzymes were observed in OVX group followed by reversing effect in treatment groups enabling to understand that bone mineralizing nutrients and minerals seem to show positive effect on ovariectomy induced oxidative damage.
\end{abstract}

Keywords: Basic bone mineralizing nutrients, genestein, postmenopausal osteoporosis, Reactive oxygen species (ROS), Vitamin K2,

Abbreviations: Reactive oxygen species (ROS), Bone mineral density (BMD), Malondialdehyde (MDA), Catalase (CAT), Superoxide dismutase (SOD), Reduced Glutathione (GSH).

\section{Introduction}

Biological aging is a normal physiological process of life and continues till death. Like every other organ system of the body, skeletal homeostasis is maximized in the first half of life. Bone remodeling slows down as age advances, especially in the case of women where estrogen deficiency after menopause and aging go hand in hand leading to multiple forms of stress as well as increased susceptibility to numerous diseases [1]

Loss of ovarian hormone estrogen following menopause, progressively disturbs the balance between bone formation and resorption [2] [3]. As estrogen receptors are present on bone forming osteoblast lineage, its deficiency leads to loss of signaling cascade required for osteoblast activation and decreased bone formation. Although old age is considered to be a dominant factor contributing to bone loss, many studies have shown strong correlation between estrogen loss, oxidative stress and osteoporosis [4] [5]. Oxidative stress resulting from an increase in intracellular ROS is the major determinant of aging. This increase in free radical overwhelms the natural antioxidant defense mechanism, subjecting individual to hyper oxidant stress. Estrogen loss and increased ROS directly targets the bone mass by enhancing transforming growth factor (TGF), limiting $\mathrm{CD}_{4}{ }^{+} \mathrm{T}$ cell activation and producing proinflammatory cytokines IL1, IL6 and TNF $\alpha$, in effect leading to RANKL activation and osteoclastogenesis [5] [6]. The universal prescription of Calcium, Vitamin D and Zinc does not address estrogen deficiency and ROS in postmenopausal woman, so also the associated inflammation and matrix mineralization.

\subsection{Objective:}

We formulated nutrient supplementation taking above factors into consideration. Antiosteoporotic treatment including other nutritional supplements along with dietary natural antioxidant would be more beneficial in improving BMD and related antioxidant status. Present study is based on a combination therapy that directly targets ROS, inflammation, estrogen deficiency and matrix mineralization in ovariectomized rats. In this study we investigated stress due to ROS production post ovariectomy and then amelioration by a combination of treatments containing basic bone mineralizing nutrients, Genestein and Vitamin K2.

\section{Materials and methods:}

All chemicals used in the study were of analytical grade and procured from the local market. The study was approved by institutional animal ethics committee (IAEC).

2.1 Animals: The adult female (180-200g) wistar rats (NINstrain) were purchased from National institute of nutrition, Hyderabad, India. They were housed in cages ( 3 per cage) and allowed to acclimatize for a period of 
two weeks, twenty rats were ovariectomized, five were SHAM operated and five rats were treated as normal control.

2.2 Ovariectomy procedure: The method of Barlet et al was followed to perform dorso-lateral ovariectomy [7]. Briefly, the rats were anesthetized with intra-peritoneal injection of thiopentane $(45 \mathrm{mg} / \mathrm{kg}$ body weight). Bilateral ovariectomy was performed by giving a small incision dorso-laterally on both sides below the rib cage. Ovaries were located, excised and openings were sutured. In SHAM operation, ovaries were exposed but not excised.

2.3 Treatments: The animals were allowed 15 days recovery period post surgery. They were carefully monitored for water, feed intake and body weight. Osteoporotic changes were observed after three months and oral treatment was then started. Rat dose for nutrients and minerals was calculated by toxicokinectic scaling [8]. Various oral treatments (Table 1) were formulated. Pilot experiments were performed to determine the daily night intake of feed per cage and supplements were added accordingly. The treatments were continued for three and a half months.

Table1: Details of various treatments used in the study.

\begin{tabular}{|c|c|c|c|c|c|}
\hline $\begin{array}{l}\text { Sr. } \\
\text { no }\end{array}$ & Treatment Group & $\mathrm{n}$ & Ovariectomy/ SHAM & \multicolumn{2}{|c|}{$\begin{array}{l}\text { Nutrient supplement } \\
\text { (Dosage Concentration in per } \mathrm{Kg} \text { of Body wt) }\end{array}$} \\
\hline 1 & OVX & 5 & Ovariectomy & \multicolumn{2}{|l|}{ None } \\
\hline 2 & SHAM & 5 & SHAM operated & \multicolumn{2}{|l|}{ None } \\
\hline \multirow[t]{7}{*}{3} & \multirow[t]{7}{*}{$\mathrm{T} 1$} & \multirow[t]{7}{*}{5} & \multirow[t]{7}{*}{ Ovariectomy } & L-Lysine & $93.75 \mathrm{mg}$ \\
\hline & & & & L-Arginine & $208.33 \mathrm{mg}$ \\
\hline & & & & L-Proline & $46.875 \mathrm{mg}$ \\
\hline & & & & L-Ascorbic Acid & $93.75 \mathrm{mg}$ \\
\hline & & & & Calcium carbonate & $250 \mathrm{mg}$ \\
\hline & & & & Zinc sulphate & $5.8 \mathrm{mg}$ \\
\hline & & & & Vitamin D3 & $100 \mathrm{IU}$ \\
\hline 4 & $\mathrm{~T} 2$ & 5 & Ovariectomy & \multicolumn{2}{|c|}{ T1+62mg Genistein Isoflavone complex } \\
\hline 5 & T3 & 5 & Ovariectomy & \multicolumn{2}{|l|}{$\mathrm{T} 2+12 \mathrm{mg}$ vitamin $\mathrm{K} 2$} \\
\hline 6 & $\mathrm{CON}$ & 5 & No Operation & \multicolumn{2}{|l|}{ none } \\
\hline
\end{tabular}

\section{Preparation of bone and liver samples:}

At the end of oral treatment, rats were sacrificed by $\mathrm{CO}_{2}$ asphyxiation. Both liver and femur bone were excised, trimmed of connective tissues and separately collected in phosphate buffered saline and Tris $\mathrm{HCl}$ respectively $(\mathrm{pH}=7.4)$. They were immediately homogenized and centrifuged at $5000 \mathrm{rpm}$ for 5 minutes. Supernatant thus obtained was used in biochemical analysis.

\subsection{Biochemical analysis:}

3.1.1 Lipid peroxidation: Malondialdehyde (MDA) was measured spectrophotometrically in the liver and bone supernatant in terms of thiobarbituric acid reactive substances [9]. It is a measure of lipid peroxidation.

3.1.2 Catalase: Catalase (CAT) was measured in liver and bone supernatant. The oxidative carbonyl modification of proteins was evaluated spectrophotometrically and the amount of $\mathrm{H}_{2} \mathrm{O}_{2}$ decomposed per minute was calculated from molar extinction co-efficient of hydrogen peroxide [10].

3.1.3 Superoxide dismutase (SOD): SOD was measured in the supernatant. The ability of enzyme to inhibit auto oxidation of pyragyllol in the presence of EDTA and was used as a measure of SOD activity. One unit of enzyme activity described as 50\% inhibition of auto oxidation of pyragyllol, as determined by the change in absorbance per minute at 420nm [11]. The activity of SOD expressed as units/mg protein.

3.1.4 Tissue Glutathione (GSH): Glutathione in liver and bone tissue was calculated using GSH standard and results are reported in nano grams GSH per ml of supernatant [12].

Stastical analysis: Analyse-it software version 2.26 was used to analyze the result.

\section{Results and Discussion}

Oxidative stress resulting from the production of ROS damages several biomolecules including lipids, proteins and DNA. Increasing evidence suggests that oxidative stress is responsible for pathophysiological process of aging and several age related diseases. In case of osteoporosis, oxidative damage diminishes bone formation by reducing the differentiation and survival of osteoblasts and enhances ROS dependent activation of osteoclast. 


\subsection{Treatments T1, T2 and T3:}

4.1.1 T1: The prescribed bone mineralizing supplements usually contains Calcium, Vitamin D and Zinc. We expanded it to contain L- Lysine, L-Proline, L-Arginine and L-Ascorbic acid. L-Lysine, L-Proline and LAscorbic acid have been shown to enhance synthesis of collagen I, major constituent of extracellular matrix [13].

4.1.2 T2: T1 was further expanded by adding genestein isoflavones, a phytoestrogen as a hormone supplement for postmenopausal estrogen deficiency.

4.1.3 T3: $\mathrm{T} 2$ was further supplemented with vitamin $\mathrm{K} 2$, a key vitamin promoting the carboxylation step of bone formation.

\subsection{Ovariectomy induced changes:}

4.2.1 Body and uterus weight: Ovariectomy in female wistar rats leads to deposition of fat and increase in body weight. A significant increase (approximately 71.8\%) in body weight was observed in OVX group over SHAM and CON. There was $75 \%$ reduction in weight of uterus in OVX group as compared to SHAM and CON. Histology of uterus revealed atrophy in some areas (data not given). Similar results have been reported by many workers [14].

\subsubsection{Ovariectomy induced oxidative stress and antioxidant status in liver and bone:}

Oxidative stress and antioxidant status was determined in both liver and bone to evaluate the overall and localized status respectively. We thought that bone would give more appropriate and location specific picture [2]. In liver, the oxidative stress (MDA) and antioxidant parameters (SOD, CAT and GSH) did not differ significantly in OVX when compared with CON and SHAM (Table 2). OVX group demonstrated increased lipid peroxidation with $26.8 \pm 11.1 \mathrm{~nm} / \mathrm{mg}$ protein as compared to CON showing $21.9 \pm 3.6 \mathrm{~nm} / \mathrm{mg}$ protein and SHAM with $20.1 \pm 3.0 \mathrm{~nm} / \mathrm{mg}$ protein. Antioxidant parameters in both liver and bone were decreased but the effect was more pronounced in bone. In bone, MDA was significantly high in OVX over CON and SHAM. Antioxidant parameters in SHAM group (both liver and bone) showed some stress when compared to CON, but it was not significant. SOD and GSH decreased significantly from CON group after ovariectomy, but did not show significance when compared with SHAM.

Table 2: Changes in oxidative stress and antioxidant status as a result of ovariectomy in female Wistar rats.

\begin{tabular}{|c|c|c|c|c|c|c|}
\hline & \multicolumn{3}{|c|}{ Liver $(n=5)$} & \multicolumn{3}{|l|}{ Bone $(n=5)$} \\
\hline & $\mathrm{CON}$ & SHAM & OVX & $\mathrm{CON}$ & SHAM & OVX \\
\hline \multicolumn{7}{|l|}{ Oxidative stress } \\
\hline $\begin{array}{l}\begin{array}{l}\mathrm{MDA} \pm \mathrm{SE} \\
\text { protein) }\end{array} \\
\end{array}$ & $21.9 \pm 3.6$ & $20.1 \pm 3.0$ & $26.8 \pm 11.1$ & $100.4^{\mathrm{b}} \pm 29.2$ & $86.9^{\mathrm{b}} \pm 44.5$ & $241.17^{\mathrm{a}} \pm 23.5$ \\
\hline \multicolumn{7}{|l|}{ Antioxidant parameters } \\
\hline $\mathrm{SOD} \pm \mathrm{SE}(\mathrm{U} / \mathrm{mg}$ of protein) & $0.97 \pm 0.2$ & $0.6 \pm 30.2$ & $0.6 \pm 0.13$ & $1.18^{\mathrm{b}} \pm 0.3$ & $0.59^{\mathrm{ab}} \pm 0.18$ & $0.26^{\mathrm{a}} \pm 0.09$ \\
\hline $\mathrm{GSH} \pm \mathrm{SE}(\mathrm{ng} / \mathrm{ml})$ & $5.3 \pm 1.8$ & $4.1 \pm 0.8$ & $3.47 \pm 0.6$ & $8.5^{\mathrm{b}} \pm 1.4$ & $5.4^{\mathrm{ab}} \pm 2.0$ & $0.26^{\mathrm{a}} \pm 0.28$ \\
\hline $\begin{array}{ll}\mathrm{CAT} \pm \mathrm{SE} & \\
(\mathrm{umoles} \quad \text { of } & \mathrm{H}_{2} \mathrm{O}_{2} \\
\text { decomposed } / \mathrm{min} / \mathrm{mg} & \text { of } \\
\text { protein) } & \end{array}$ & $0.93 \pm 0.1$ & $0.7 \pm 0.08$ & $0.75 \pm 0.08$ & & & \\
\hline
\end{tabular}

Different superscript indicates that values differ significantly.

$\mathrm{n}=$ No. of animals per group.

In conclusion, OVX group showed increased lipid peroxidation and decreased antioxidant status. SHAM operation also caused some stress and showed decreased antioxidant parameters. Our results are in agreement with Maggio et al. [15]. SHAM and CON did not show any significant difference in both the tissues. 4.3 Effect of treatments T1, T2 and T3 on ovariectomy induced oxidative stress and antioxidant status in liver and bone.

All the three treatments, $\mathrm{T} 1, \mathrm{~T} 2$ and $\mathrm{T} 3$ contained ascorbic acid and zinc as major oxidant scavengers. MDA levels recorded a decrease from $26.8 \pm 11.1 \mathrm{~nm} / \mathrm{mg}$ protein in OVX to $20.6 \pm 10$ in $\mathrm{T} 1$ and $20.5 \pm 4.6$ in $\mathrm{T} 2$ (figure $1)$. 
Figure 1: Malondialdehyde activity in ovariectomized Wistar rats.
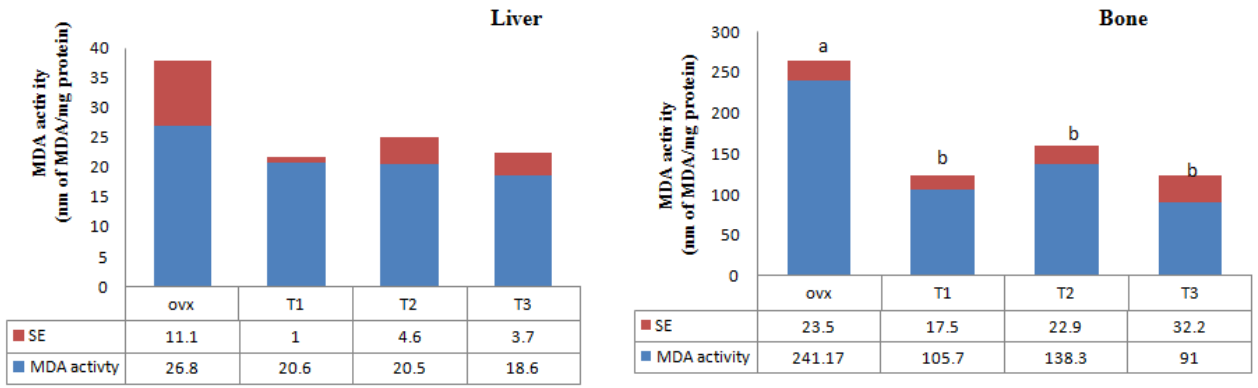

Figure 1: Effect of oral treatments T1, T2 and T3 on Malondialdehyde activity in ovariectomized Wistar rats (OVX)

Addition of genestein to T1 (T2) did not have any effect on lipid peroxidation in liver. A further decrease to $18.6 \pm 3.7$ was observed when $\mathrm{T} 2$ was supplemented with Vitamin K2. In bone, all treatments significantly reduced the MDA activity when compared with OVX group, but T3 values showed best results followed by $\mathrm{T} 1$ and $\mathrm{T} 2$. As liver activity is the overall effect of tr eatment, addition of genestein to T1 did not result in any change, but at localized levels genestein recorded about 33\% increase in T2 over T1. Antioxidant parameter, SOD was highest in $\mathrm{T} 3$ in liver showing an increase from $0.6 \pm 0.05 \mathrm{U} / \mathrm{mg}$ protein in OVX to $1.18 \pm 0.3 \mathrm{U} / \mathrm{mg}$ protein in T3. (Figure 2).

Figure 2: Superoxide dismutase activity in ovariectomized Wistar rats.
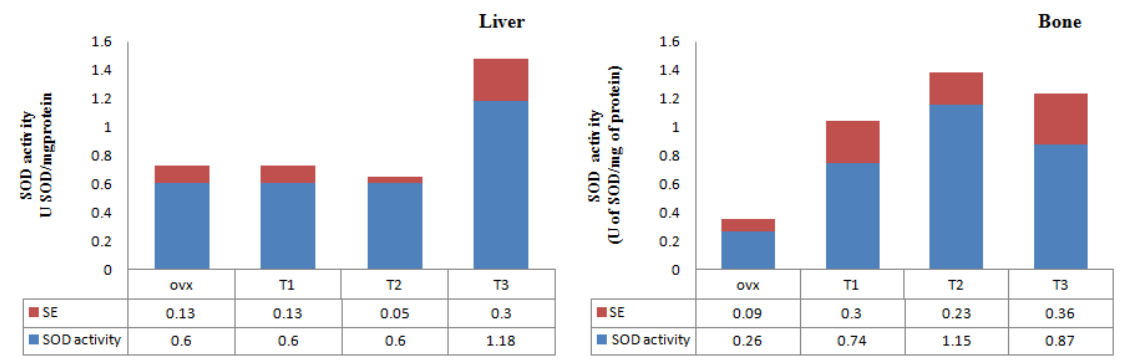

Figure 2: Effect of oral treatment T1, T2 and T3 on Superoxide Dismutase activity in ovariectomized Wistar rats (OVX)

T2 showed highest activity in bone and T3 was most effective in liver. Both T1 and T2 recorded no change in SOD activity. This probably indicates the effect of Vitamin K2 in T3. Reduced glutathione (GSH) content also showed a differential effect in liver and bone. In liver, T1 and T3 led to increased GSH over OVX i.e. from $3.47 \pm 0.6 \mathrm{mg} / \mathrm{ml}$ in OVX to $4.8 \pm 1.6 \mathrm{mg} / \mathrm{ml}$ and $4.3 \pm 1.1 \mathrm{mg} / \mathrm{ml}$ respectively, but $\mathrm{T} 2$ (genestein supplementation) actually resulted in decrease in GSH. In bone, T1 was most effective followed by T2 and T3, (Figure 3).

Figure 3: Reduced glutathione activity in ovariectomized Wistar rats.

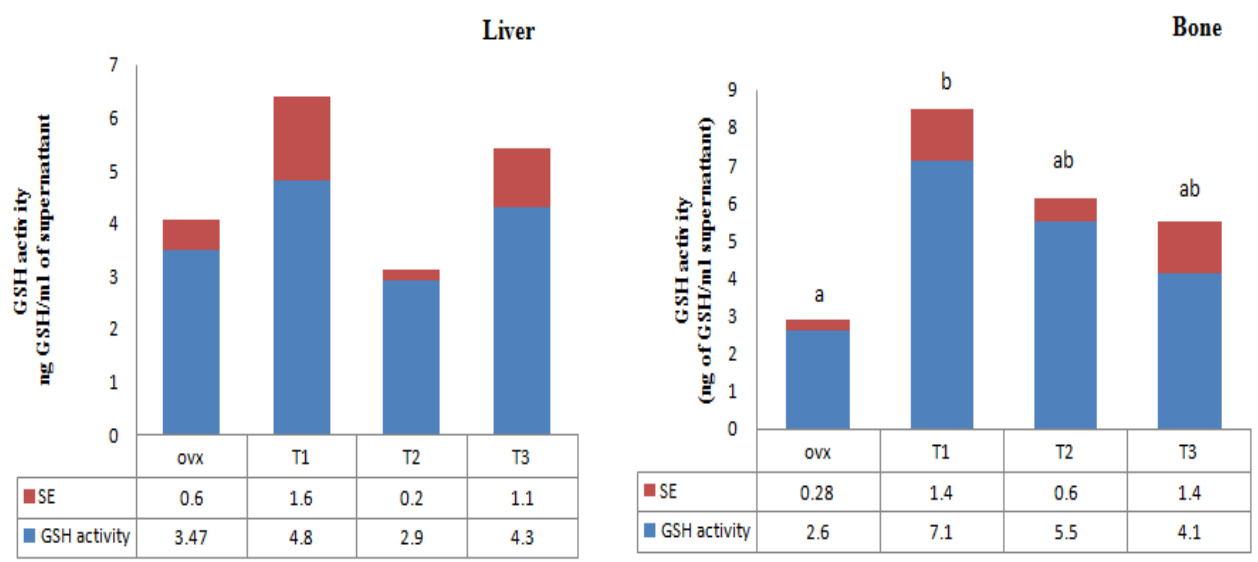

Figure 3: Effect of oral treatments T1, T2 and T3 on Reduced Glutathione activity in ovariectomized Wistar rats (OVX). 
All the treatments resulted in significant increase in GSH in bone tissue over OVX. CAT is another important antioxidant enzyme which is reported to be low in OVX [16]. In liver all the three treatments increased CAT activity significantly over OVX but highest was recorded in T3 showing an increase from $0.75 \pm 0.08$ in OVX to $1.19 \pm 0.15$ in T3 (Figure 4). SOD and CAT works hand in hand as $\mathrm{H}_{2} \mathrm{O}_{2}$, a product of SOD activity is acted upon by CAT [17]. SOD and CAT are more pronounced in T2 and T3. In spite of repeated experiments, we could not detect CAT activity in bone supernatant.

Figure 4: Catalase activity in ovariectomized Wistar Rats.

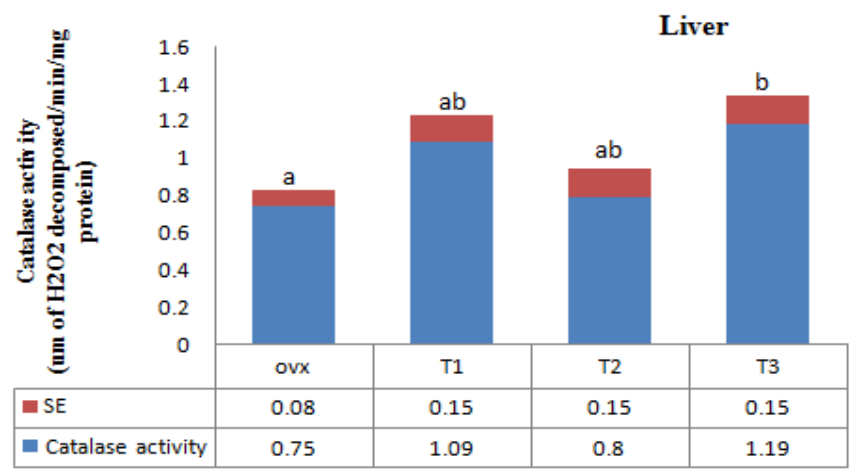

Figure 4: Effect of oral treatments $\mathrm{T} 1, \mathrm{~T} 2$ and $\mathrm{T} 3$ on Catalase activity in ovariectomized Wistar rats (OVX)

Increased deposition of MDA in OVX over SHAM and CON, in both liver and bone and significant decrease in antioxidant enzyme SOD, GSH and CAT clearly indicates that ovariectomy and estrogen deficiency has caused oxidative stress and has lowered antioxidant status in ovariectomized rats [18]. This imbalance between oxidative stress and antioxidant agents leads to enhanced osteoclast activity and inhibition of osteoblast cells in bone. NADPH oxidase in osteoclast cells generates cytokine regulated ROS. ROS are potent inducers of various factors that are implicated in bone loss and estrogen deficiency. Thus loss of hormone estrogen that is responsible for oxidant defenses in bone is contributing to oxidative stress [3]. MDA was lowered in our Treatment group T1 T2 and T3 in both liver and bone indicating diminished osteoclast activity in bone and increased functioning of antioxidant enzymes. L- Ascorbic acid and Zinc in T1 along with bone mineralizing nutrients is helping in improved antioxidant status. L-Ascorbic acid acts as a central component of antioxidant machinery and Zinc is known as a cellular antioxidant, it is active at cellular levels through induction of metallothionines, a group of low molecular weight amino acids, the production of which is induced by zinc in major tissues including liver and kidney [19]. Also Morton et al showed that ascorbic acid when supplemented with calcium and estrogen improved BMD [20]. Decrease in MDA was followed by elevated SOD activity in bone and in liver with one fold increase in T3. Zinc regulates the levels of extracellular form of superoxide dismutase which destroys superoxide radical and protects against nitric oxide mediated free radical formation. Studies have reported low level of zinc in osteoporotic conditions and further its supplementation was able to make up the loss [21]. Increase in SOD in T3 could be explained due to synergestic effect of Genestein and Vitamin K. Genestein is reported to enhance bone mineralization in invitro [22]. Combination treatment of genestein and ascorbic acid is also reported by Hall SL et al [23]. This observation is supported by Borras et al, showing up regulation of SOD gene by genestein isoflavone [24]. Vitamin K, a fat soluble quinone does not act directly as an antioxidant but once absorbed and located in the cell membranes it is involved in several pathways of antioxidant defense system. A study conducted by Sakomoto et al on aged albino Wistar rats have shown that even higher doses of vitamin K2 supplements was not able to improve antioxidant status. [25]. But our observations shows vitamin K2, when supplemented with our formulations results in decreased oxidant status and this synergy is contributing to reduced oxidative stress in T3. Our results probably point to the better management with vitamin K2 supplementation (T3) as indicated by lowest MDA activity. Also many studies have shown positive correlation between vitamin $\mathrm{K}$ supplementation, bone formation and improved BMD [26]. Decrease in GSH levels is the marker of oxidative stress. Increase in GSH level over OVX was the result of our treatments in bone and liver. Ascorbic acid is the major component responsible for increasing cellular levels of GSH and its recycling. Increase in GSH could be explained in terms of fate of glutathione in oxidatively challenged cells. Also additional physiological function of ascorbic acid is to maintain active sites of metal ions which keep the enzyme in reduced state [27]. And it was found that ascorbic acid is responsible for increasing cellular levels of glutathione even in GSH deficient animals [28]. As GSH is constantly being converted into oxidized and reduced form in the tissue, looking into either of these forms would have been more beneficial to us. Also complete antioxidant protection requires simultaneous presence of ascorbic acid both intracellular and 
extracellular [29]. Studies conducted by three different researchers, Morton et al, Chavan et al, and Ayse asan et al have also reported lowering of oxidative stress after ascorbic acid supplementation in osteoporosis [20], [30], [31]. Invitro studies of Xiao XH et al have shown that ascorbic acid when supplemented in dose dependent manner inhibits osteoclastogenesis and stimulates osteoblast differentiation [32]. CAT has highest turnover rates of all enzymes. One molecule of catalase converts millions of molecules of $\mathrm{H}_{2} \mathrm{O}_{2}$ into water and oxygen per second. Increase in CAT activity in treatment group over OVX and decreased MDA shows liver CAT activity is enhanced in treatment group. Also increased production of superoxide ion is converted to hydrogen peroxide by SOD enzyme which in turn is cleared by CAT. Increase in SOD corresponds to increase CAT in treatment groups. Thus a constant flux of reactions is observed resulting into changes.

Aging, estrogen deficiency and oxidative stress are the contributing factors resulting into osteoporotic bone. Decreased consumption of vitamins and minerals with aging also contribute to low antioxidant status and increased oxidative stress (18], [33). Proper consumption of dietary antioxidants may play a protective role in maintaining bone health [34]. Thus casting out synergistic effect of basic bone mineralizing nutrients on osteoporotic bone, as to see which combination would be better, our results indicates $\mathrm{T} 1$ to be sufficient than the other two groups. Its synergistic effect was able to improve antioxidant status in postmenopausal condition. Also in our previous study, we found improvement in serum biomarkers (unpublished data) and trabecular bone biology [35] with supplementation of basic bone mineralizing nutrients. We conclude that as antioxidant mechanism slows down with age and proper intake of dietary formulation is beneficial in reducing stress generated by aging and estrogen deficiency and bone loss.

\section{Acknowledgement}

This work is being funded by Matthias Rath funding agency USA, the authors greatly acknowledge their financial support.

\section{References:}

[1]. M. A Sanchez-Rodriguez, M. Ruiz-Ramos, E. Correa-Munoz and V. Manuel Mendoza-Nunez, Oxidative stress as a risk factor for osteoporosis in elderly Mexicans as characterized by antioxidant enzymes, BMC Musculoskeletal Disorders, 8:124 doi: 10.1186/1471-2474-8-124 (2007).

[2]. R. Pacifici, Postmenopausal Osteoporosis: How the Hormonal Changes of Menopause Cause Bone Loss, ( $3^{\text {rd }}$ edition) Osteoporosis, (Elsevier, Inc. 2008), pg 1041-1054.

[3]. M. Almeida, L. Han, Skeletal Involution by Age-associated Oxidative Stress and Its Acceleration by Loss of Sex Steroids, Journal of biological chemistry, September 14; 282(37), (2007) 27285-27297.

[4]. J. Balasch, Sex steroids and bone: current perspectives, Human Reproduction Update, Vol.9, No.3, (2003), $207 \pm 222$.

[5]. F. Wauquie, L. Leotoin, V. Coxam, J. Guicheux and Y. Wittrant, Oxidative stress in bone remodeling and disease, Elsevier Ltd. cell press doi:10.1016/j.molmed.2009.08.004.

[6]. M. J. Steinbeck, W.H. Appel, A J. Verhoeven, M. J. Karnovsky, NADPH-Oxidase Expression and In Situ Production of Superoxide by Osteoclasts Actively Resorbing Bone, The Journal of Cell Biology,126, (1994) 765-772.

[7]. J.P. Barlet, V. Coxam, M.J. Davicco, N. Gamet, Animal models for osteoporosis, Reproduction nutrition, review, vol 34: (1994) 221-236.

[8]. K. Bachmann, D. Pardoe, D. White, Scaling Basic Toxicokinetic Parameters from Rat to Man, Environmental Health Perspectives, 104 (1996).

[9]. S.J. Dormandy, Autooxidation of Human red cell lipids induced by hydro-gen peroxide, British Journal of Hematology, 20, (95) (1971).

[10]. H. Aebi, Catalase, Methods of enzymatic analysis, Vol.3, Academic Press, New York, 3, (1983), 273.

[11]. S.L. Marklund and G. Marklund, European Journal of Biochemistry, 47, (1974):469

[12]. E. Beutler, O. Duron, B.M. Kelly, Improved method of the determination of blood glutathione. Journal of laboratory and clinical medicine, volume 61(5), (1963), 882-890.

[13]. S. Viguet-Carrin P. Garnero, P Delmas, The role of collagen in bone strength Osteoporosis International 17, (2006) $319-336$.

[14]. U. Modder, B. Riggs, T. Spelsberg, D. Fraser, E. Atkinson, R. Arnold, S. Khosla, Dose- response of estrogen on bone versus the uterus in ovariectomized mice, European Journal of endocrinology, 151, (2004) 503-510.

[15]. D. Maggio, M. Barbani, M. Pierandrei, M. Polidiri, M. Catani, P. Mecocci, U. Senin, R. Pacific, A. Cherubini, Marked Decrease in Plasma Antioxidants in Aged Osteoporotic Women: Results of a Cross-Sectional Study, The Journal of Clinical Endocrinology \& Metabolism 88(4), 2003, 1523-1527.

[16]. O.F. Sendur, Y. Turan, E. Tastaban, M. Serter, 2009, Antioxidant status in patients with osteoporosis: A controlled study, Joint Bone Spine, 76, (2009) 514-518

[17]. J. M. Lean, C. J. Jager, B. Kirstein, K. Fuller, T. J. Chambers, Hydrogen Peroxide Is Essential for Estrogen Deficiency Bone Loss and Osteoclast Formation, Endocrinology, 146 (2), (2005), 728-735.

[18]. S.C. Manolagas, From Estrogen-Centric to Aging and Oxidative Stress: A Revised Perspective of the Pathogenesis of Osteoporosis, Endocrine Reviews, June: 31(3):266-300 (2010).

[19]. R. Claeyssen, M.A. Sanchez, J. Arnaud, L. Touvard, A. Alonso, Y. Chancerelle, A.M. Roussel and D. Agay, Burn-induced oxidative stress is altered by a low zinc status: Kinetic study in burned rats fed a low zinc diet, Biological Trace element research 126, (2008), S80-S96.

[20]. D. J. Morton, 2001, Vitamin C Supplement Use and Bone Mineral Density in Postmenopausal Women, Journal of Bone and Mineral Research, Volume 16 (2001).

[21]. R.A. Romero, M.M Arcas, C. Monteagudo, G. Lopez, M. L. Lorenzo, F.M. Ocana- Peinado, F. Olea- Serrano, Association between dietary antioxidant quality score (DAQs) and bone Mineral density in Spanish women, Nutricion Hospitalaria, 27(6), (2012), 18861893. 
[22]. S. Uchiyama, M. Yamaguchi 2006, Genestein and zinc synergistically enhance gene expression and mineralization in osteoblastic MC3T3-F1 cells, International journal of molecular medicine, 9, (2007), 213-220.

[23]. S.L. Hall, The relation of dietary vitamin C intake to bone mineral density: results from the PEPI study. Calcified Tissue International 63(3), (1998), 183-9.

[24]. C. Borras, J. Gambini, M.C. Cabrera, J. Sastre, Feredico, V. Pallardo, Giovanni E. Mann \& J. Vina 2005, Genistein, a soy isoflavone, up-regulates expression of antioxidant genes: involvement of estrogen receptors, ERK1/2, and NFK $\beta$, The FASEB Journal, doi: 10.1096/fj.05-5522fje (2005).

[25]. W. Sakamoto, H. Isomura, K. Fujie, T. Lizuka, J. Nisnihira, G. Tatebe, K. Takahashi, Y. Osaki, M. Komai, H Tamai, The effect of vitamin K2 on bone metabolism in aged female rats, Osteoporosis international 16(12), (2005), 1604-10.

[26]. J. Adams, J. Pepping, 2005, Vitamin K in the treatment and prevention of osteoporosis and arterial calcification, American Journal Health System Pharmacy, 62(15), (2005) 1574-1581.

[27]. E.C. Opara and S.W. Rockway, Antioxidants and Micronutrients, Dis Mon; 52, (2006), 151-163 doi:10.1016/j.disamonth.2006.05.002 (2006).

[28]. J. Martensson, A. Meister, Proceedings of the National Academy of Sciences, USA 88, (1991) 4656-4660.

[29]. V.Montecinos, Vitamin C is an Essential Antioxidant that Enhances Survival of Oxidatively Stressed Human Vascular Endothelial Cells in the Presence of a Vast Molar Excess of Glutathione, The journal of biological chemistry, http://www.jbc.org/cgi/doi/10.1074/jbc.M608361200 (2007)

[30]. S.N. Chavan, U. More, S. Mulgund, V. Saxena and A.N. Sontakke, Effect Of Supplementation Of Vitamin C And E On Oxidative Stress In Osteoporosis, Indian Journal of Clinical Biochemistry, 22 (2), (2007), 101-105.

[31]. A. Arslan, S. Orkun, G. Aydin, I. Keles, A. Tosun, M. Arslan, O. Caglayan, Effects of ovariectomy and ascorbic acid supplement on oxidative stress parameters and bone mineral density in rats, Libyan Journal of Medicine, 6:5965-DOI: 10.3402/ljm.v6i0.5965 (2011).

[32]. XH. Xiao, EY. Liao, HD Zhou, RC. Dai, LQ. Yuan, Wu XP, Ascorbic acid inhibits osteoclastogenesis of RAW264.7 cells induced by receptor activated nuclear factor kappaB ligand (RANKL) in vitro. Journal of Endocrinology Investigation.(3), (2005), 253-60.

[33]. J.L. Wells, A.C. Dumbrell, Nutrition and aging: assessment and treatment of compromised nutritional status in frail elderly patients, Clinical interventions in aging: 1(1), (2006), 67-79.

[34]. R.L. Wolf, J.A. Cauley, M. Pettinger, R. Jackson, A. Lacroix, M. Leboff, C.E Lewis, M.C Nevitt, J.A Simon, K.L. Stone, and J. Wactawski-Wende, Lack of a relation between vitamin and mineral antioxidants and bone mineral density: results from the Women's Health Initiative, American Journal Clinical Nutrition, 82, (2005), 581- 8

[35]. S. Siriah, S. Kotwal, Effect of micronutrients on osteoblast and osteoclast activity in ovariectomized rat model of osteoporosis, Bionano Frontier ISSN 0974-0678 volume I (2012), pg217-219. 